commentary $^{1}$ and for sharing their audit results. ${ }^{2}$ I note that when they prepared their training and interventions, they advised either use of the combined oral contraceptive pill (COC) or the progestogen-only pill (POP) for the management of troublesome bleeding. Both Faculty of Sexual $\&$ Reproductive Healthcare guidance ${ }^{3}$ and our commentary ${ }^{1}$ cite the use of the COC but not the POP as the main intervention for implant bleeding. There is no evidence that the POP used in this manner is effective, even though anecdotally it is occasionally used. It is interesting that there was no improvement in implant retention in Drs Ronghe and Welsh's follow-up audit. I wonder if these results could be analysed to determine if it was those women given the POP who did not improve and whether or not there was any difference in the results depending upon which treatment was used.

Jane Dickson, MA, MFSRH

Consultant in Sexual and Reproductive Healthcare, Oxleas NHS Foundation Trust, London, UK; jane.dickson@nhs.net

Competing interests None.

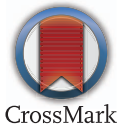

J Fam Plann Reprod Health Care 2014:40:311. doi:10.1136/jprhc-2014-101049

\title{
(S) Linked
}

http://dx.doi.org/10.1136/jprhc-2014-101034

\section{Comment on 'Unanticipated bleeding with the etonogestrel implant: advice and therapeutic interventions': author's response}

I would like to thank Drs Ronghe and Welsh for their response to our 